\title{
Exfoliating large monolayers in liquids
}

Christopher A Howard*

Department of Physics \& Astronomy, University College London, London, WC1E 6BT, UK

*c.howard@ucl.ac.uk

\section{Two-dimensional superconductors have been produced via a mild intercalation-assisted, exfoliation approach, providing large-size, high-quality single layers with the ease and versatility of liquid-phase processing.}

Many of the most exquisite properties of two-dimensional (2D) materials only occur in their monolayer form. This is notably true for transition metal dichalcogenides (TMDs) where single layers break inversion symmetry, resulting in the valley degree of freedom and unconventional superconductivity ${ }^{1,2}$. Excitingly, the interplay between such exotic effects (and those that arise due to interfacial interactions) can be explored and controlled by creating devices from interleaved 2D sheets ${ }^{2,3,4}$. Such experiments require large-area, single-crystal samples with a low density of defects and clean interfaces between adjacent layers that can be readily manipulated into devices. However, for producing 2D materials a frustrating dichotomy exists. Methods that produce large, high-quality monolayers, such as mechanical exfoliation (the 'scotch tape method') or chemical vapour deposition, are relatively pain-staking and must typically be followed by intricate transfer methods for device fabrication. On the other hand, exfoliating layered crystals in liquids is cheaper, more scalable, and has the in-built bonus that the resulting dispersions can be used to manipulate the nanosheets at high volume and low cost, for example, by ink-jet printing 5 . But while these liquid-dispersed samples have flooded the marketplace, liquid phase exfoliation (LPE) typically requires chemically or physically aggressive methods yielding materials that are not only too small and defective for devices, but only a few percent of which are monolayers, compromising the treasured properties of $2 \mathrm{D}$ materials. Writing in Nature Materials, Jing Li and co-workers ${ }^{6}$ present a electrochemical exfoliation method that delivers the best attributes of these different production approaches, and expansively demonstrate both its effectiveness in 
producing high-quality 2D superconductor monolayers, and its versatility for different processing technologies.

The authors exfoliate 2D superconductor monolayers using intercalation, the process of inserting arrays of guest species within the van der Waals gaps of layered materials. Intercalation finds ubiquitous technological use, being the process upon which lithium-ion batteries are based, and is typically driven by charge transfer between the guest species and the layers. Intercalation has previously been used to facilitate LPE ${ }^{5,7-10}$, but producing single-layer sheets of large size and high quality proves to be challenging. Alkali-intercalated layered materials have been shown to be spontaneously soluble in polar solvents ${ }^{7,8}$, but while this charge can be exploited for functionalisation or electroplating, it renders the solutions air-sensitive and the dissolved sheets can be relatively small ${ }^{7,8}$. High charge transfer can also induce an undesirable structural phase change within the layers ${ }^{9}$ and previous efforts to negate this, based on intercalation of larger ions, produce mainly multilayer sheets ${ }^{10}$. The approach proposed by Li and coworkers hinges on co-intercalating large cations (such as tetrabutylammonium) alongside neutral solvent molecules using electrochemical methods. This process results in large layer separations, uniform intercalation, and a relatively small charge transfer, weakening bonding between layers to gently delaminate large flakes of the crystal into the liquid. It is the close control of a dilute concentration of ions that permits tuning the process for homogenous intercalation but with a low overall charge transfer ${ }^{6}$ to yield air-stable dispersions containing predominantly monolayer sheets.

The method is demonstrated for seven different TMDs with a particular focus on $\mathrm{NbSe}_{2}$ for which monolayers of up to $300 \mu \mathrm{m}$ across are produced (Figure 1a). Low defect-density is demonstrated using scanning transmission electron microscopy and the sheets' single-crystallinity confirmed by measuring identically-aligned electron diffraction pattens across the sample. However, the 'acid test' is in the resulting device performance. The samples duly demonstrate impressively low residual resistivity values and exhibit the hallmarks of 2D superconductivity particularly the substantially enhanced upper critical field $\left(\mathrm{H}_{\mathrm{c} 2}\right)$ - the applied field up to which superconductivity survives ${ }^{1}$. The authors show the practicality of their methods by depositing the 2D superconductors directly from liquids for device creation (Figure 
1b). Remarkably, this processing takes place in air, whereas normally stringent measures are required to prevent degradation of the air-sensitive $\mathrm{NbSe}_{2}$ sheets. The authors speculate adsorbed solvent molecules protect the layers during processing, but upon stacking vacate the interface via a self-cleaning effect. The cleanliness of the interface and potential of the method for creating heterostructure devices is impressively proven by measuring a device created from two overlapping, rotated $\mathrm{NbSe}_{2}$ layers, the magneto-transport behaviour of which is consistent with a Josephson junction array with a period that matches that of the inter-sheet moiré pattern. Finally, the authors modify their process for inkjet printing. For this application, huge sheets are unsuitable since they do not fit through the nozzle, and can reduce concentrations to below those required for printing. Using the inks the authors deposit thin film structures with 2D-superconductor characteristics and in another interesting demonstration, mix with resin to 3D-print superconducting Merlion statues (Figure 1c).

New methods for producing 2D materials are eagerly received by the community, especially since it is widely acknowledged that different production routes suit different applications and studies. For example, multi-layers produced in bulk quantities may be unsuitable for devices but can be preferable for battery electrodes and composites. Other originally-projected applications are currently unexplored or impractical given the quality and/or volume of material required, and industry now focuses on value-added to final performance rather than hype of incorporated materials. In this context, methods that produce high-quality monolayers are particularly welcome since it is frustrating when the transformative potential of $2 \mathrm{D}$ materials is dismissed due to experiments with inferior or multilayer samples. Large monolayers that can be easily manipulated for devices are now required for the burgeoning field of twisted and aligned homo/heterostructures, where control over different interactions and the interfacial effects on the excitons is rapidly revealing exciting new science ${ }^{2,3,4}$. Given that multiple overlapping crystals can be produced on one substrate in ambient conditions from one drop of dispersion, it is here that this method is likely to have most immediate impact. 
a

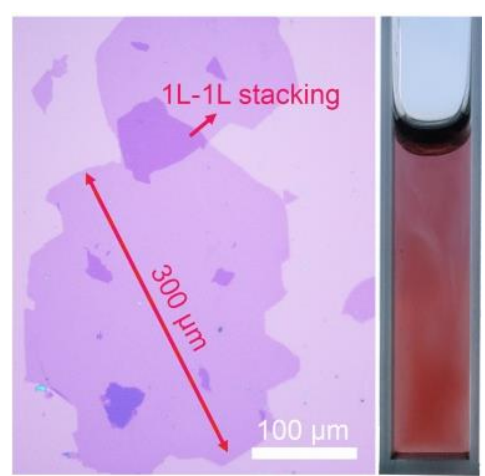

b

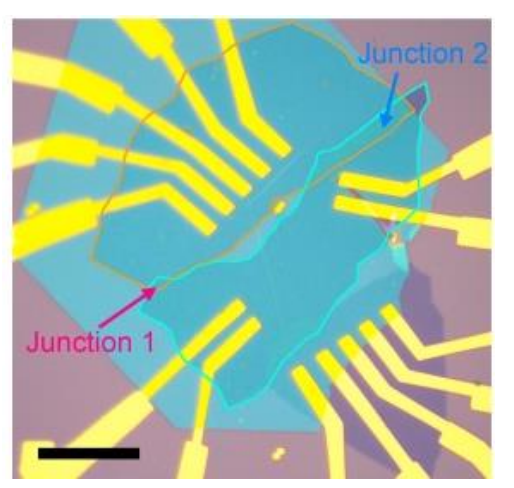

C

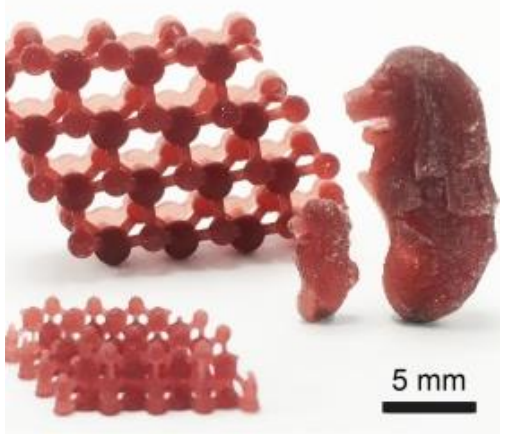

Figure 1: Devices and structures from solutions of exfoliated 2D

superconductors. a, Large-area, low-defect $\mathrm{NbSe}_{2}$ monolayers (optical micrograph, left) dispersed in liquids (photograph, right) produced by intercalation-assisted mild electrochemical exfoliation method. Such liquids can be used to process the 2D superconductors in ambient conditions to create stacked, twisted $\mathrm{NbSe}_{2}$ homojunction devices shown in $\mathbf{b}$, and to 3D print superconducting $\mathrm{NbSe}_{2}$ atomic models and the Merlion statues shown in the digital photograph c. Figure adapted with permission from ref. ${ }^{6}$, Springer Nature Ltd.

\section{References}

[1] Xi, X. et al. Nature Phys. 12, 139-143 (2016)

[2] Tran, K. et al. Nature 567, 71-75, (2019)

[3] Cao Y. et al. Nature 556, 43-50 (2018)

[4] Alexeev E. M. et al. Nature 567, 81-86 (2019)

[5] Nicolosi, V., Chowalla, M., Kanatzidis, M. G., Strano, M. S. \& Coleman, J. N.

Science 340, 1226419 (2013)

[6] Li, J. et al. Nat. Mater. https://doi.org/10.1038/s41563-020-00831-1 (2020).

[7] Vallés, C. et al. J. Am. Chem. Soc. 130, 15802-15804 (2008)

[8] Cullen, P. L. et al. Nat. Chem. 9, 244-249, (2017)

[9] Eda, G. et al. Nano Lett. 11, 5111-5116 (2011)

[10] Lin Z., et al. Nature 562, 254-258 (2018) 